\title{
Microphysics-based black carbon aging in a global CTM: constraints from HIPPO observations and implications for global black carbon budget
}

Cenlin He et al.

Correspondence to: Cenlin He (cenlinhe@atmos.ucla.edu)

The copyright of individual parts of the supplement might differ from the CC-BY 3.0 licence. 


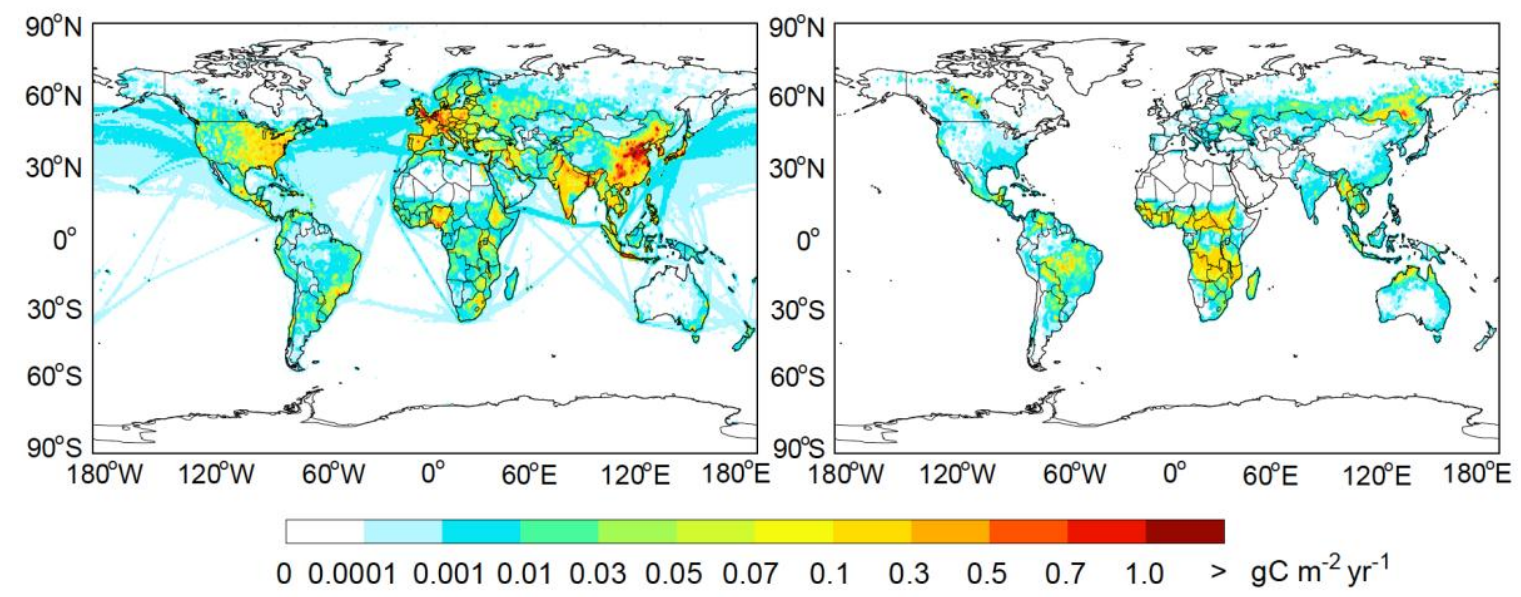

Figure S1. Global annual mean BC anthropogenic emissions from the PKU-BC inventory (left panel) and biomass burning emissions from the GFED3 inventory (right panel) for 2008. 

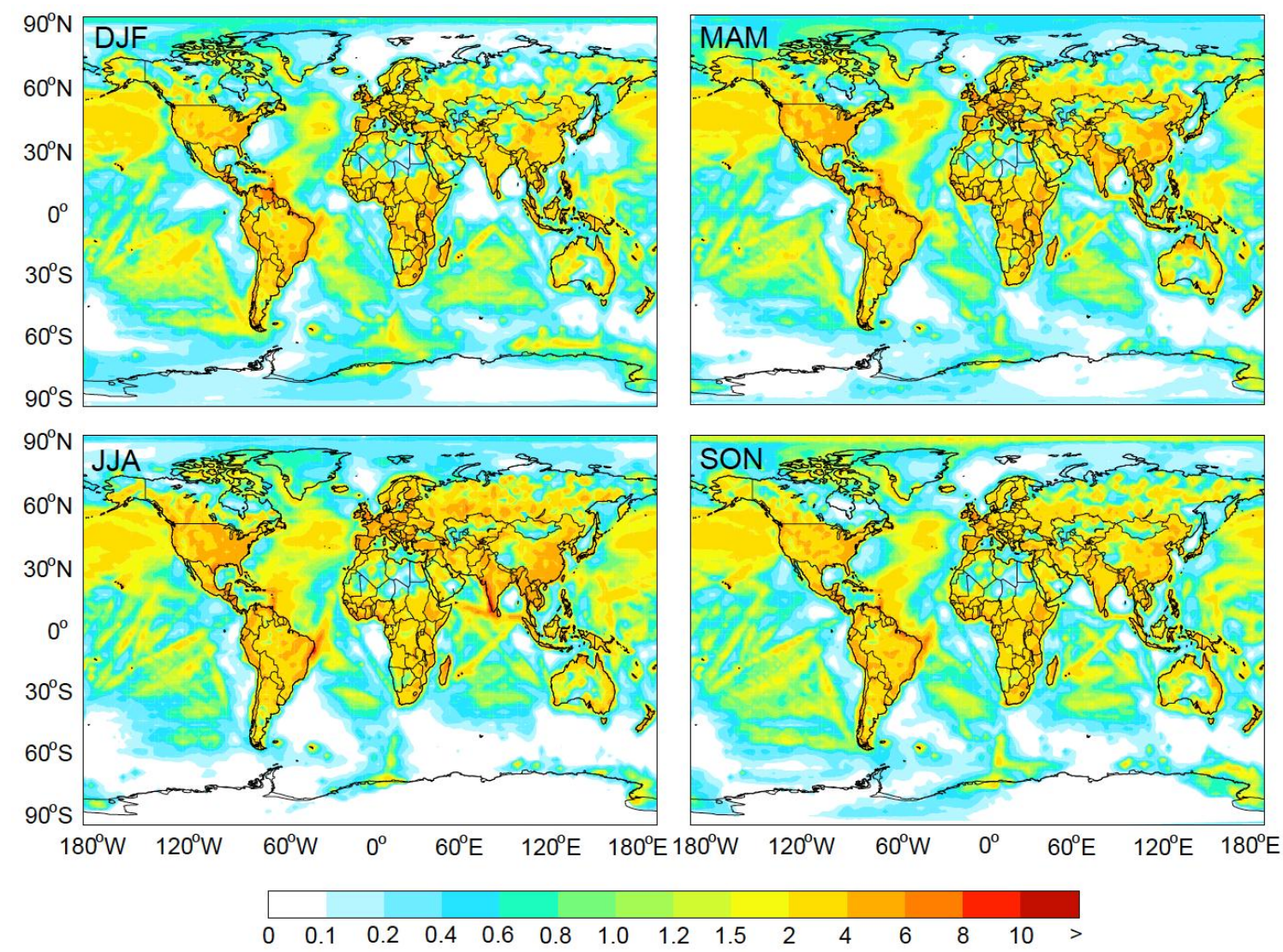

Figure S2. GEOS-Chem simulated ratios of BC aging rate from a microphysics-based scheme to a fixed aging scheme at the surface for winter (DJF), spring (MAM), summer (JJA), and fall (SON) in 2009. 


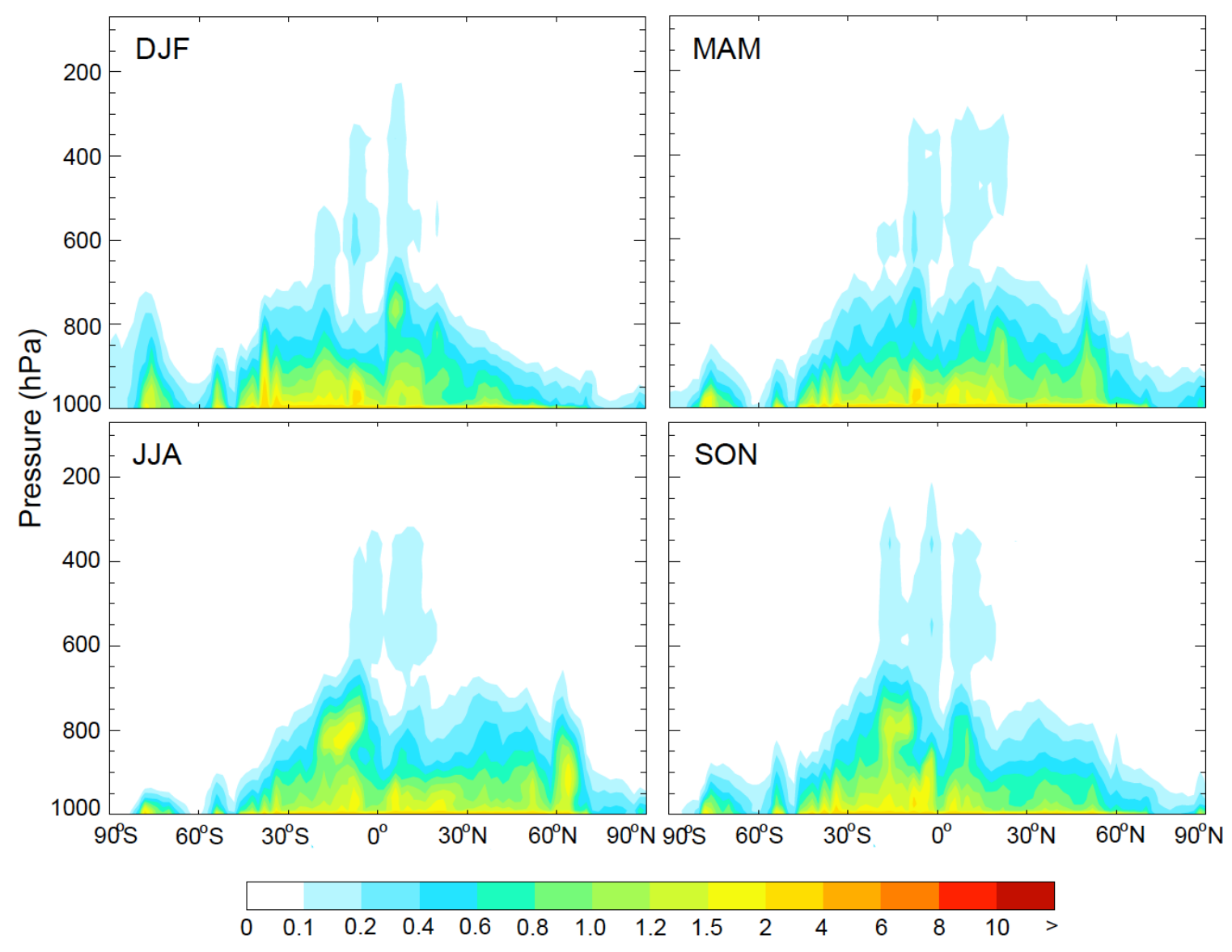

Figure S3. Same as Fig. S2, but for zonal mean ratios. 


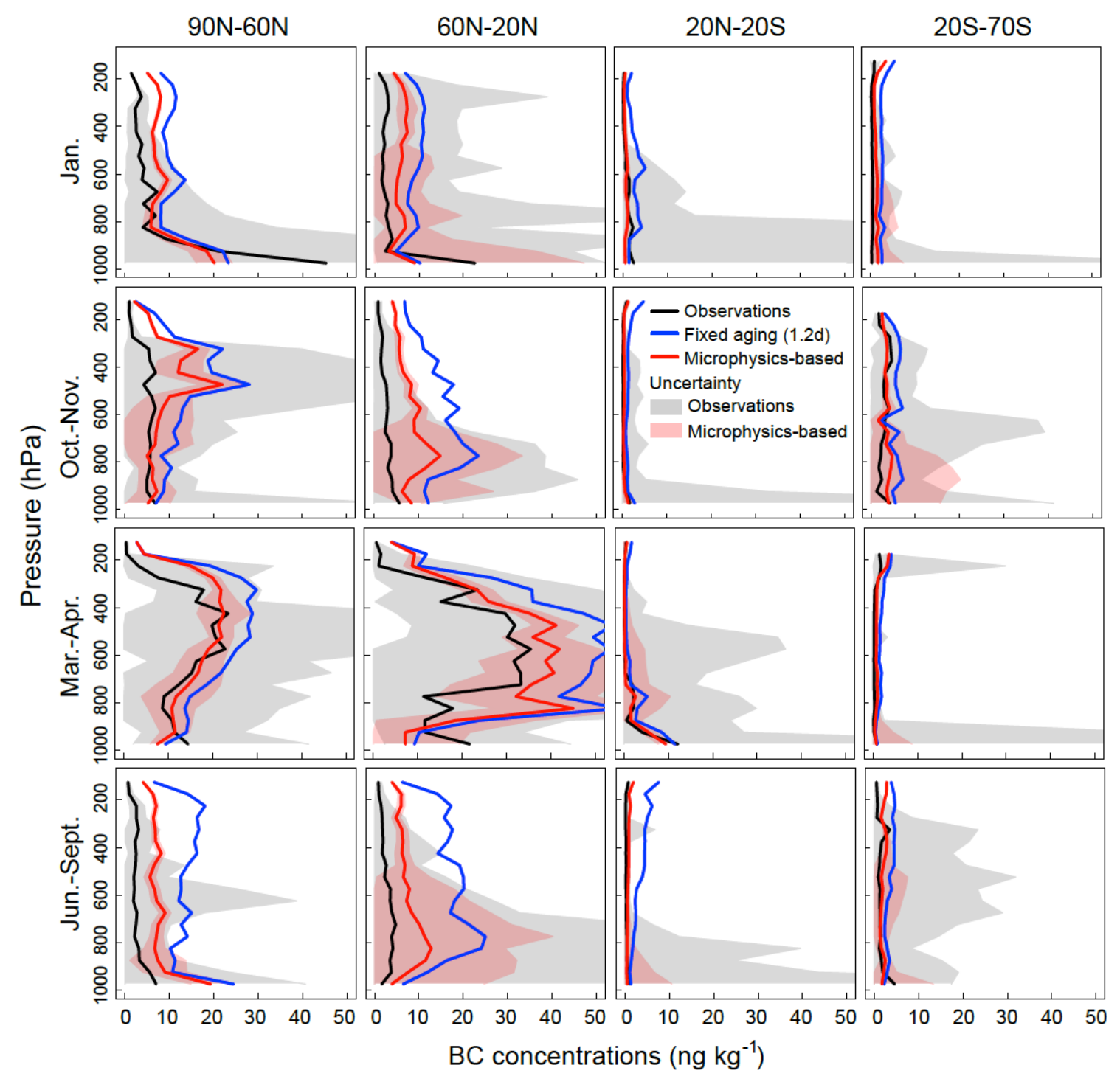

Figure S4. Median vertical profiles of HIPPO observed (black) and GEOS-Chem simulated BC concentrations at different seasons and latitudes. Results are averaged over $50 \mathrm{hPa}$ altitude bins. Model results using a fixed $\mathrm{BC}$ aging (blue) and a microphysics-based aging (red) are shown. Also shown are the 1- $\sigma$ uncertainties of observations (grey) and model results (light red) from microphysics-based sensitivity simulations (see Table 1 and text for details). 


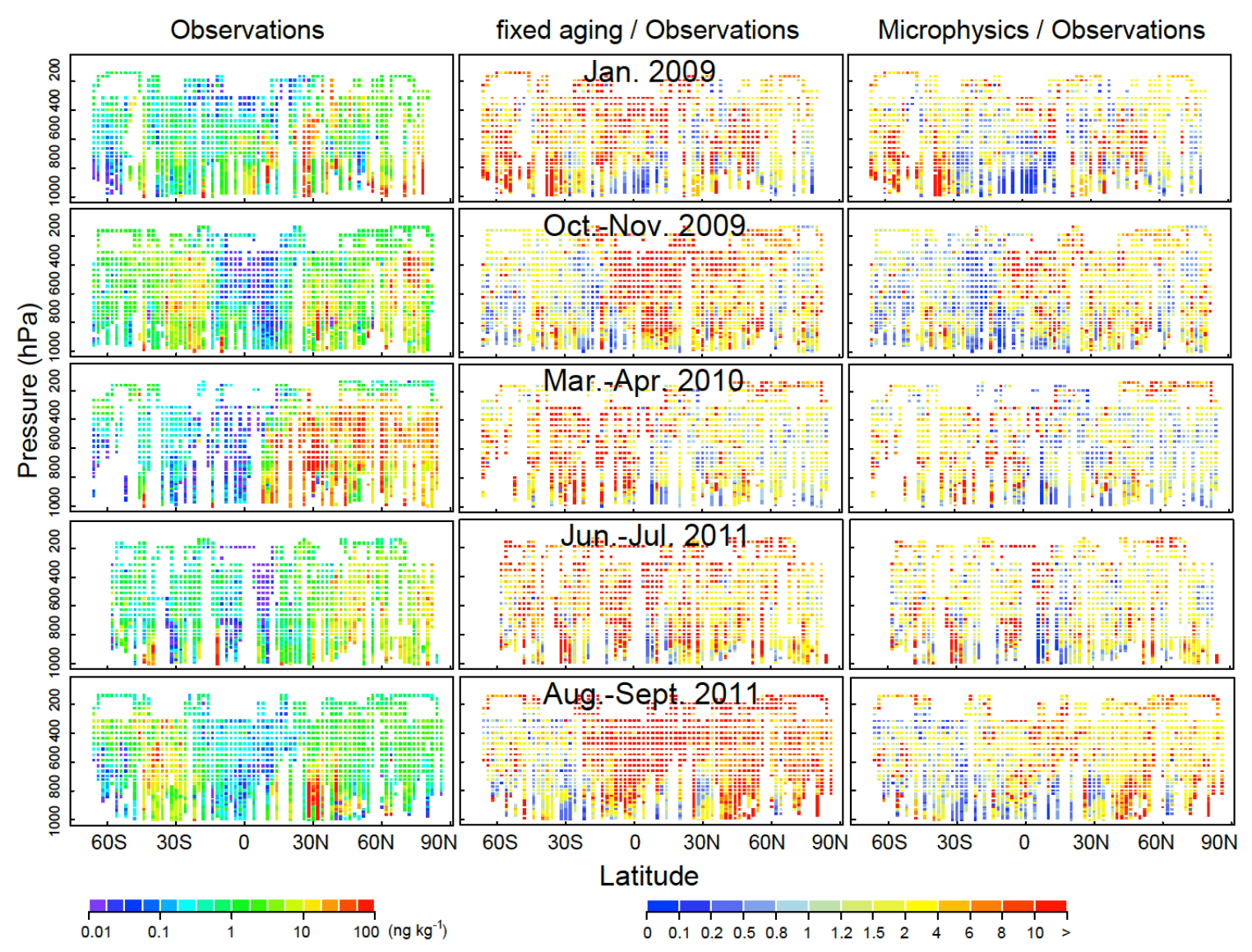

Figure S5. HIPPO observed BC concentrations at different altitudes and latitudes (left panels), ratios of GEOS-Chem simulated BC concentrations using a fixed BC aging scheme to HIPPO observations (middle panels), and ratios of GEOS-Chem simulated BC concentrations using a microphysics-based BC aging scheme to HIPPO observations (right panels). 

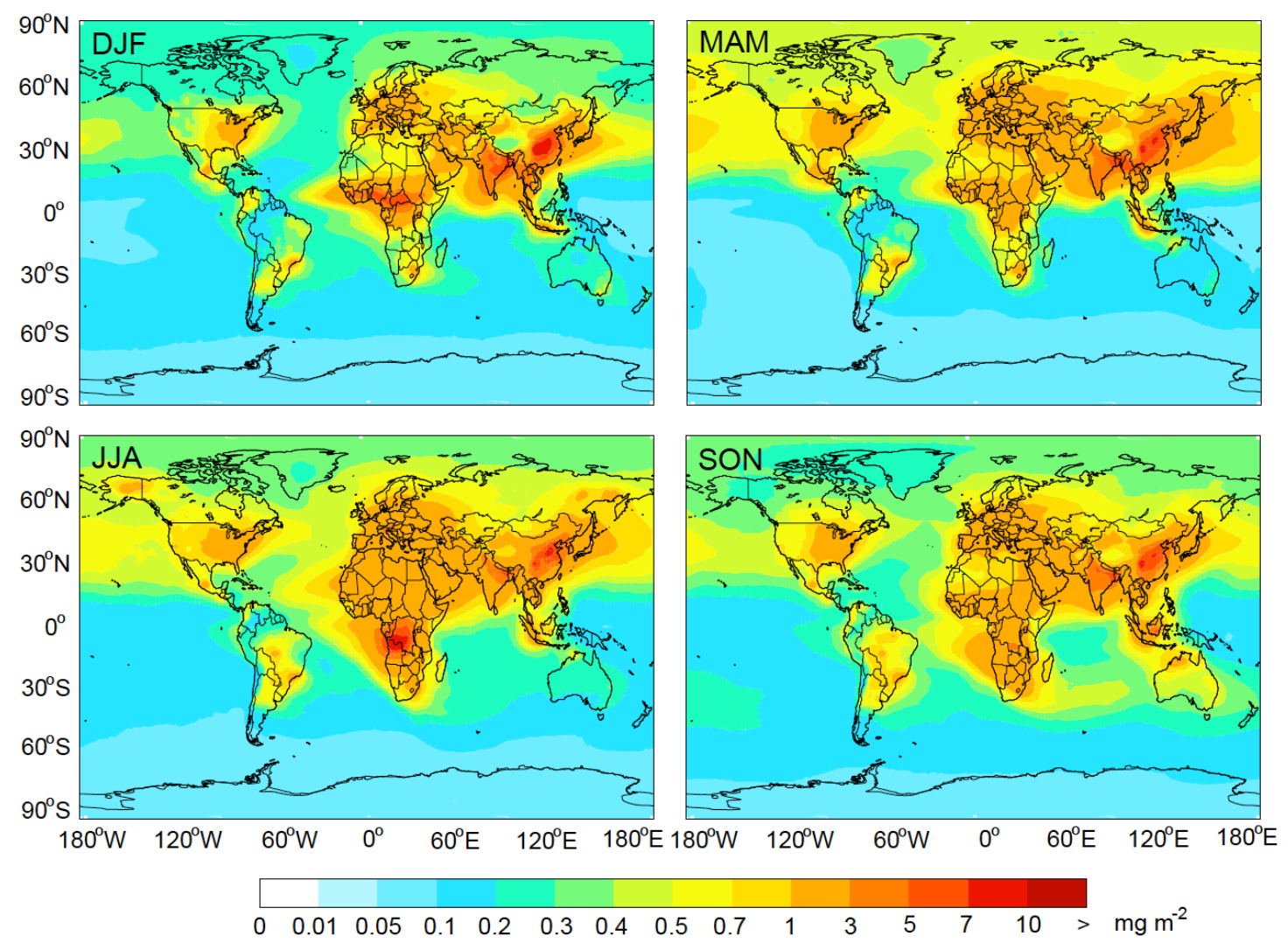

Figure S6. GEOS-Chem simulated differences of BC column burden $\left(\mathrm{mg} \mathrm{m}^{-2}\right)$ using a fixed BC aging scheme and a microphysics-based aging scheme (FIX minus MP STD, $_{\text {, see }}$ Table 1) for winter (DJF), spring (MAM), summer (JJA), and fall (SON) in 2009. 

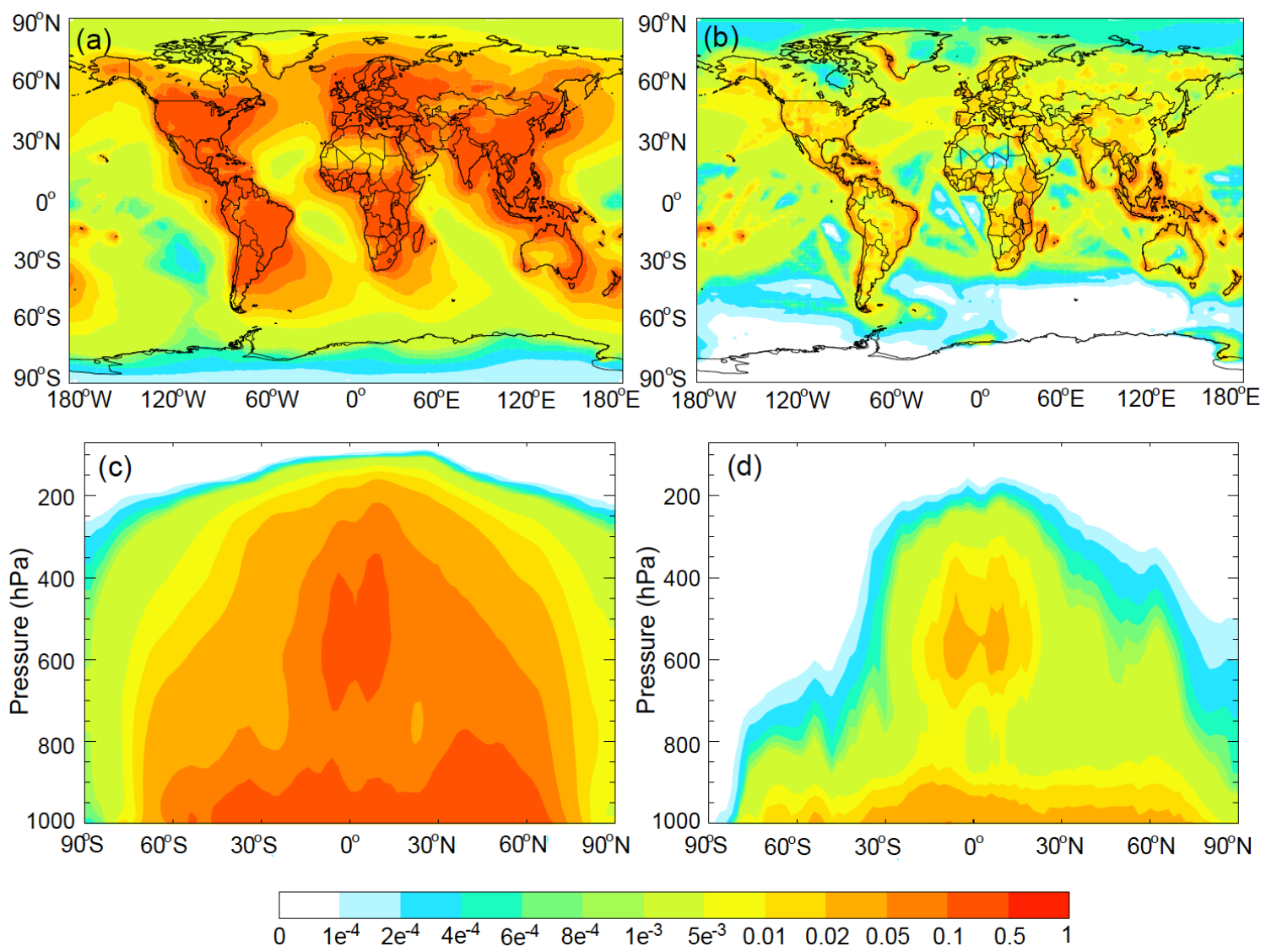

Figure S7. GEOS-Chem simulated annual mean fraction of hydrophobic BC concentration in total $\mathrm{BC}$ concentration at the surface (top panels) and zonal averages (lower panels) using a fixed BC aging scheme (left panels) and a microphysics-based aging scheme (right panels). Model results are for 2009. 


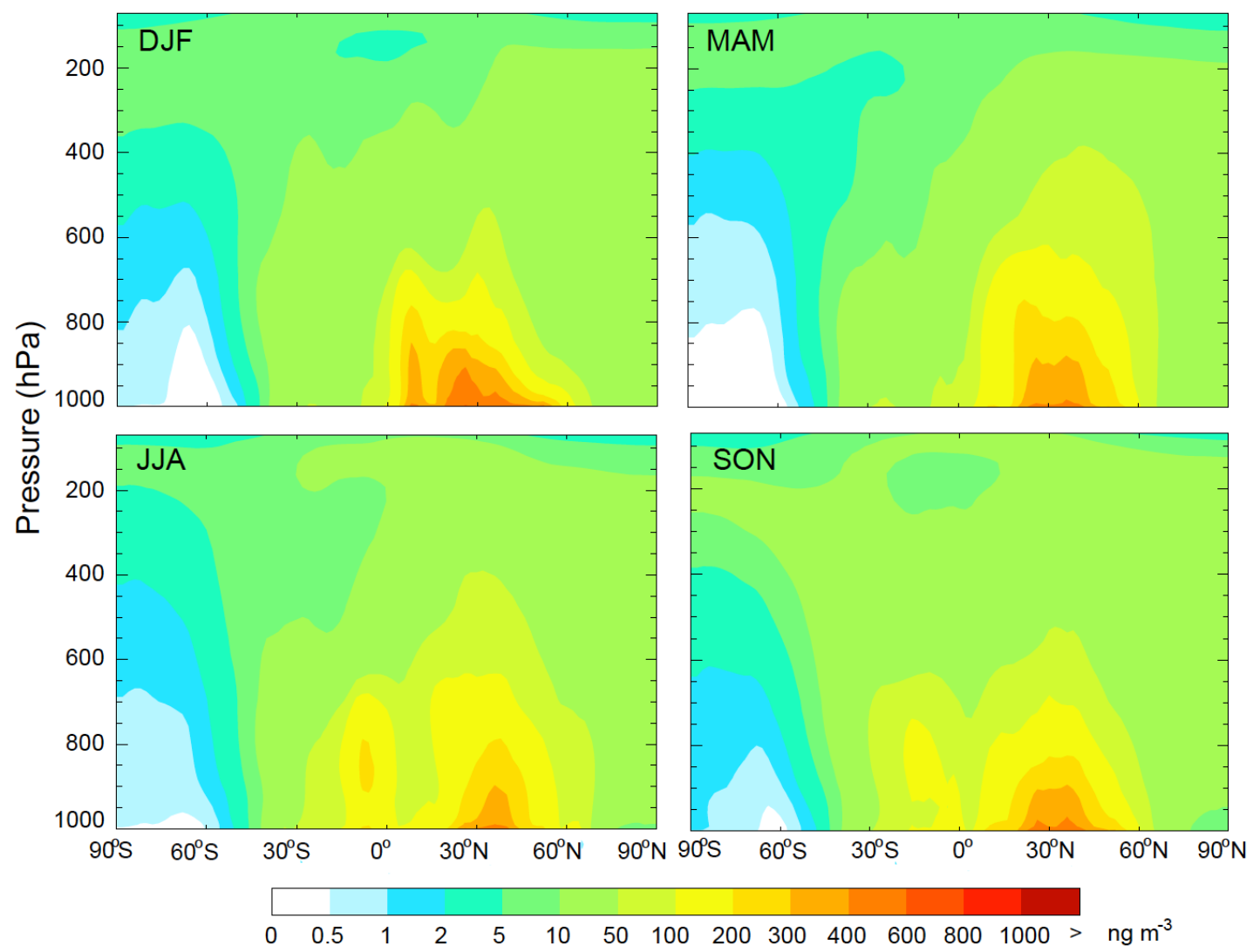

Figure S8. Same as Fig. S6, but for differences of zonal mean BC concentrations. 

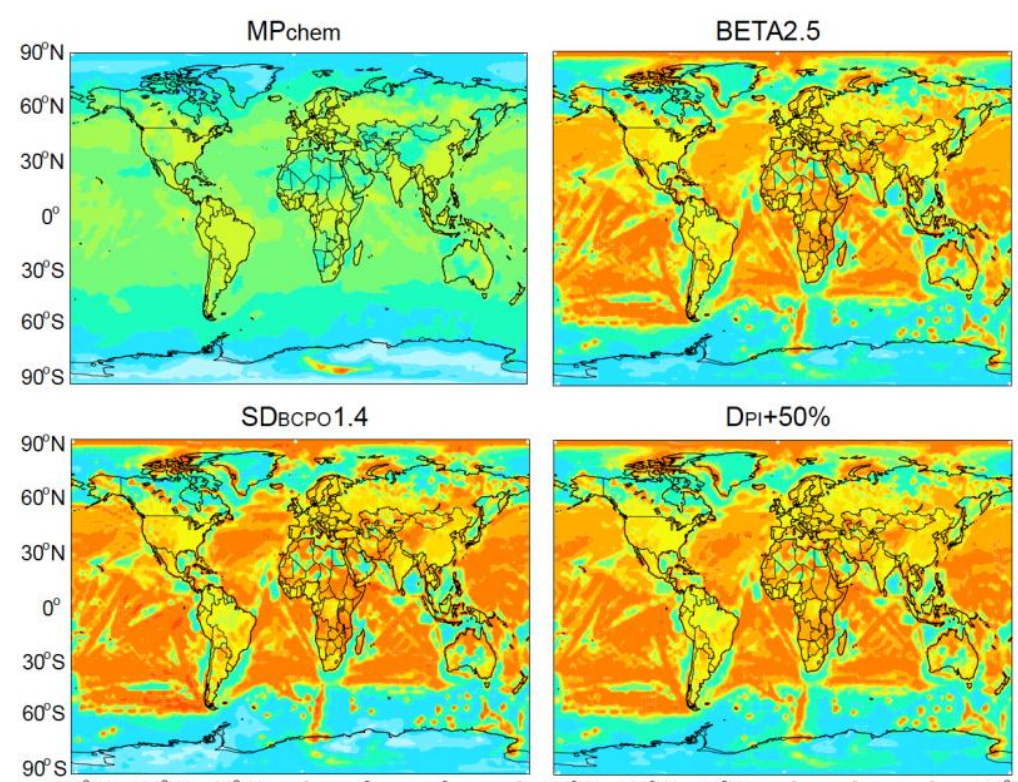

$\mathrm{DPI}+50 \%$

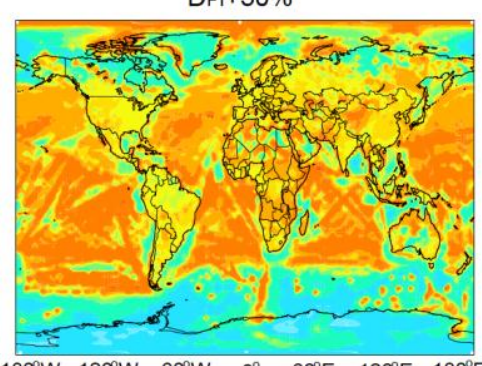

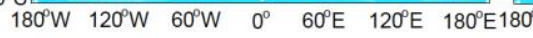

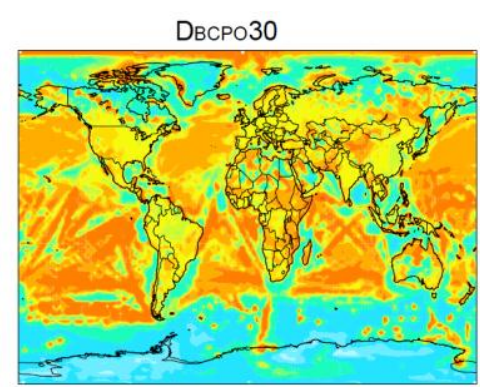

$\mathrm{SDPI}+0.2$

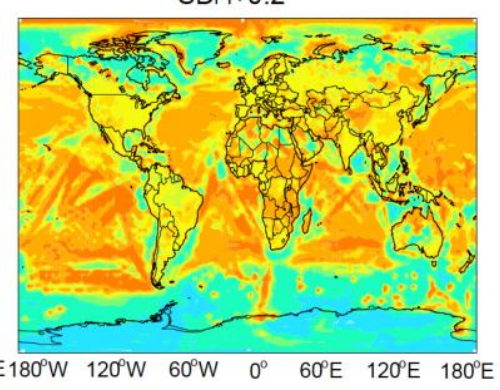

$25>$

Figure S9. GEOS-Chem simulated ratios of annual mean $\mathrm{BC}$ aging rates through condensation from six microphysics-based sensitivity simulations (Table 1) to that from the standard microphysics-based simulation in the surface layer. See Table 1 and text for details. Model results are for 2009. 


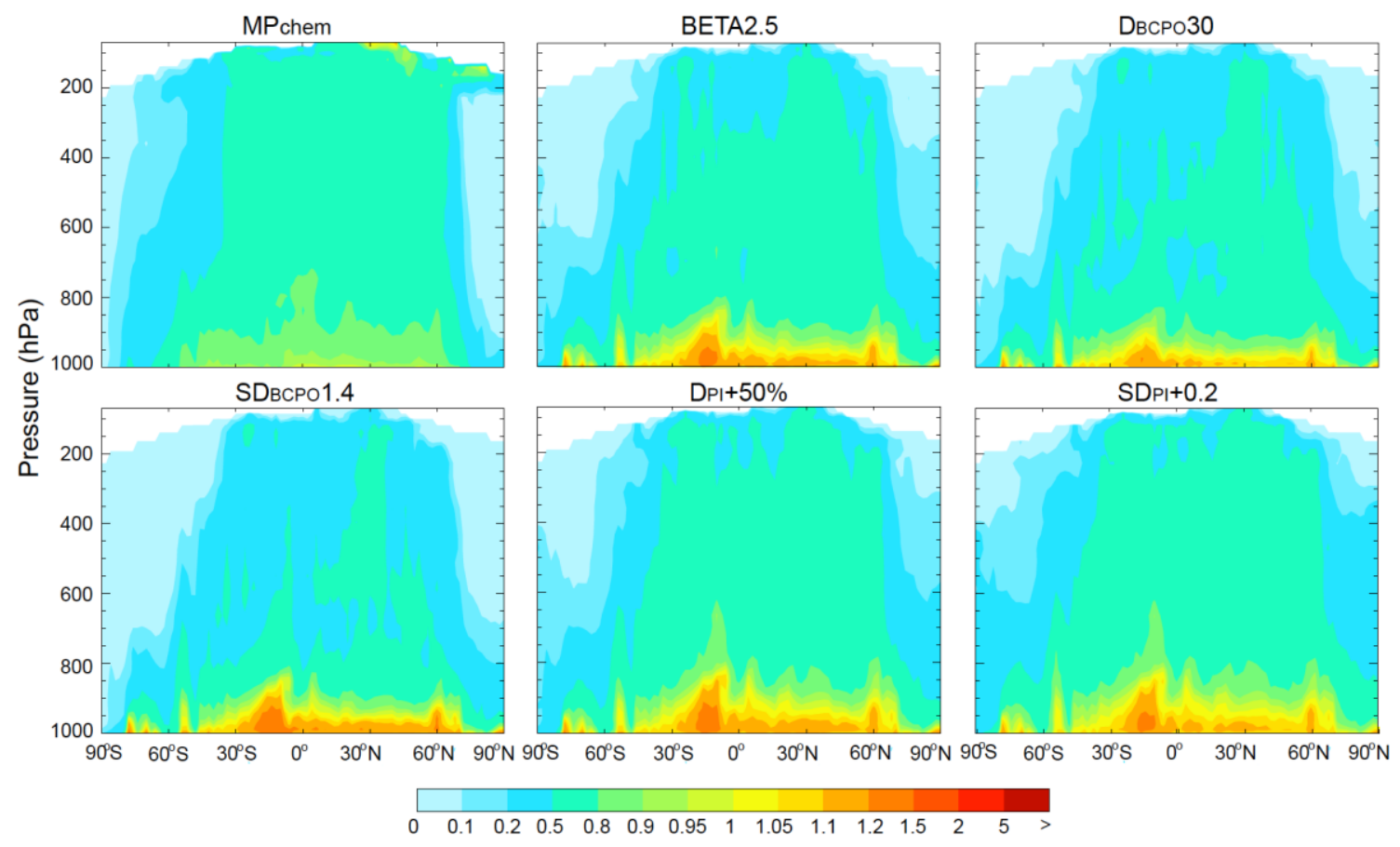

Figure S10. Same as Fig. S9, but for zonal mean ratios. 

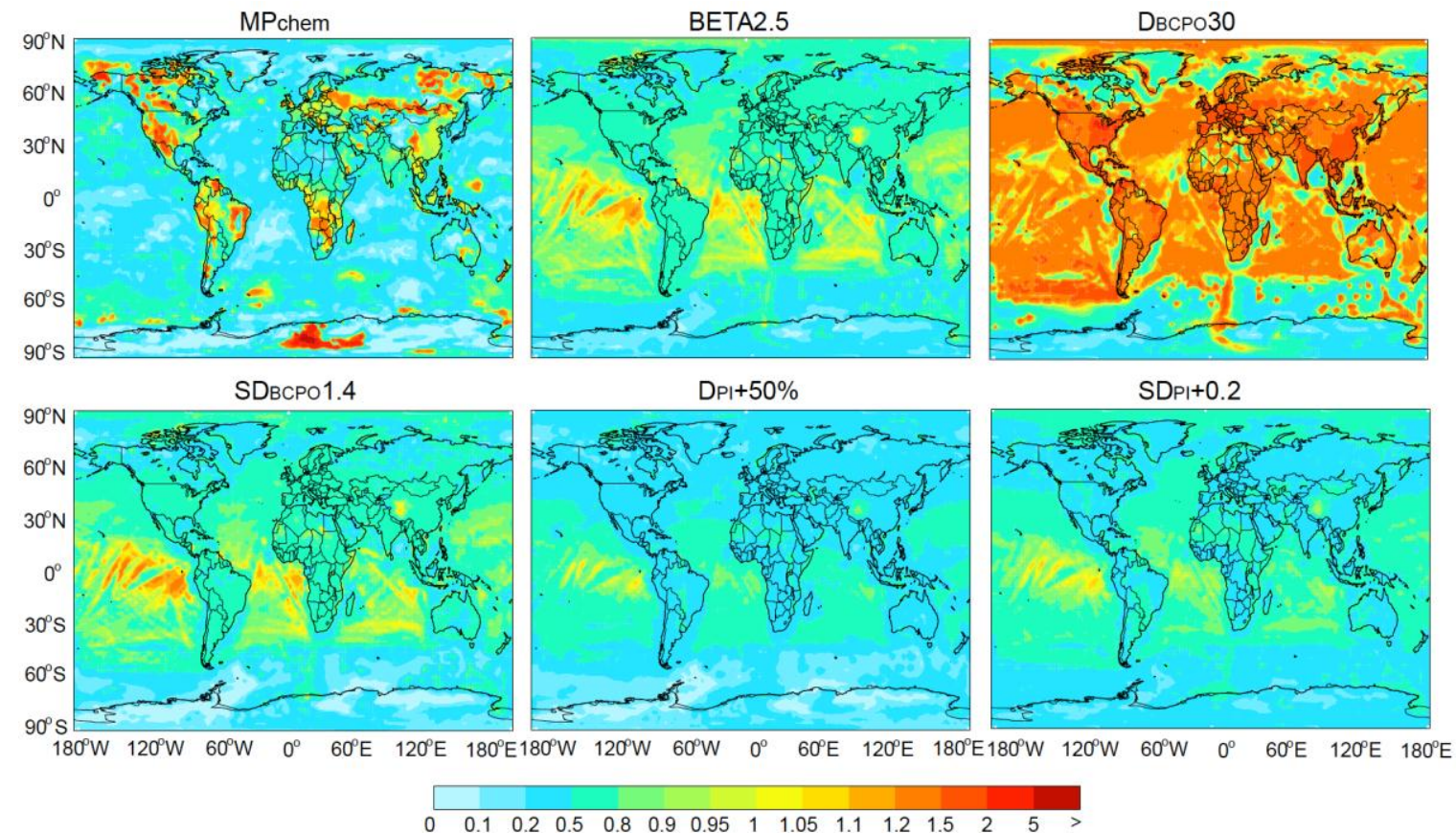

Figure S11. Same as Fig. S9, but for ratios of BC aging rates through coagulation. 


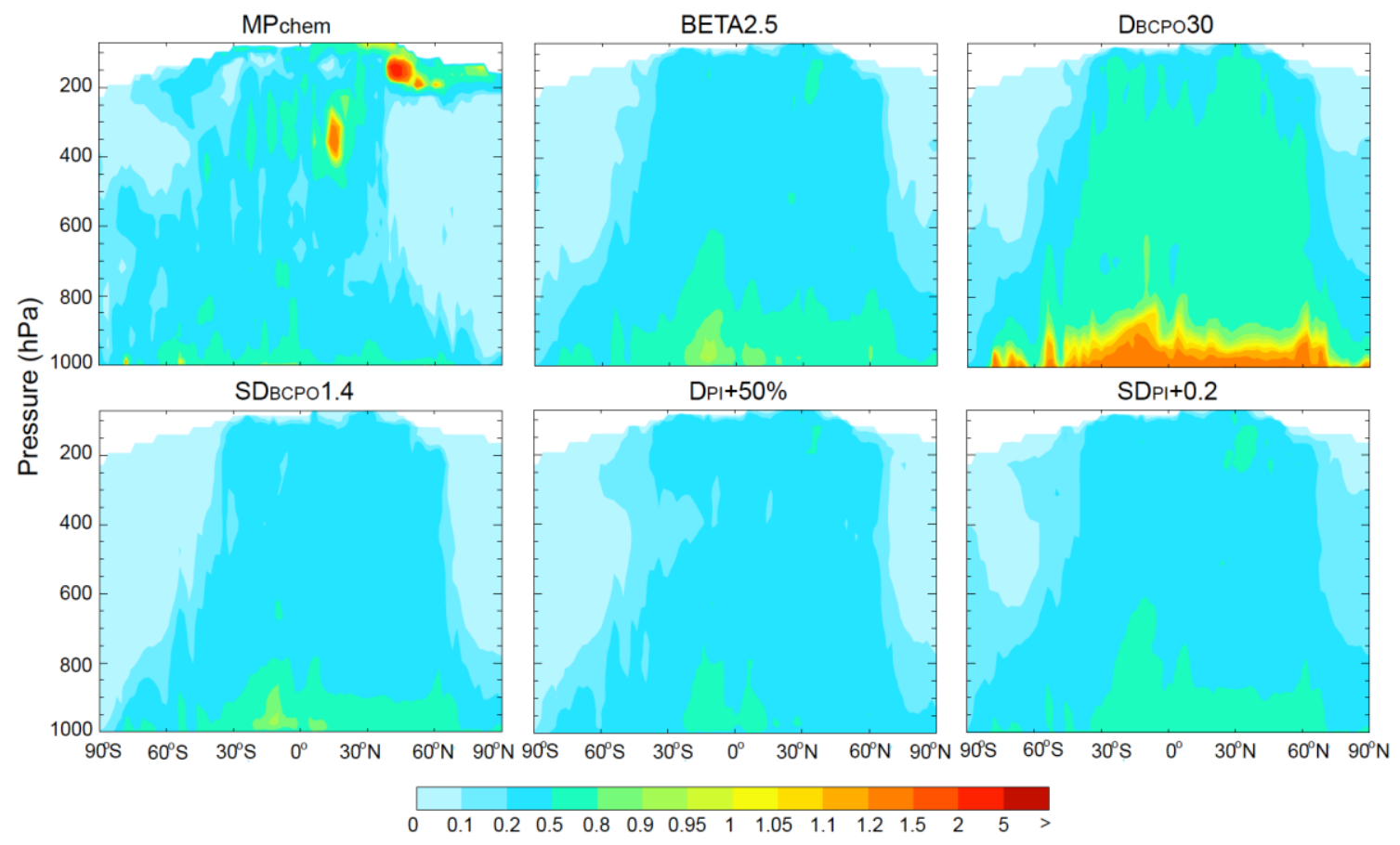

Figure S12. Same as Fig. S11, but for zonal mean ratios. 

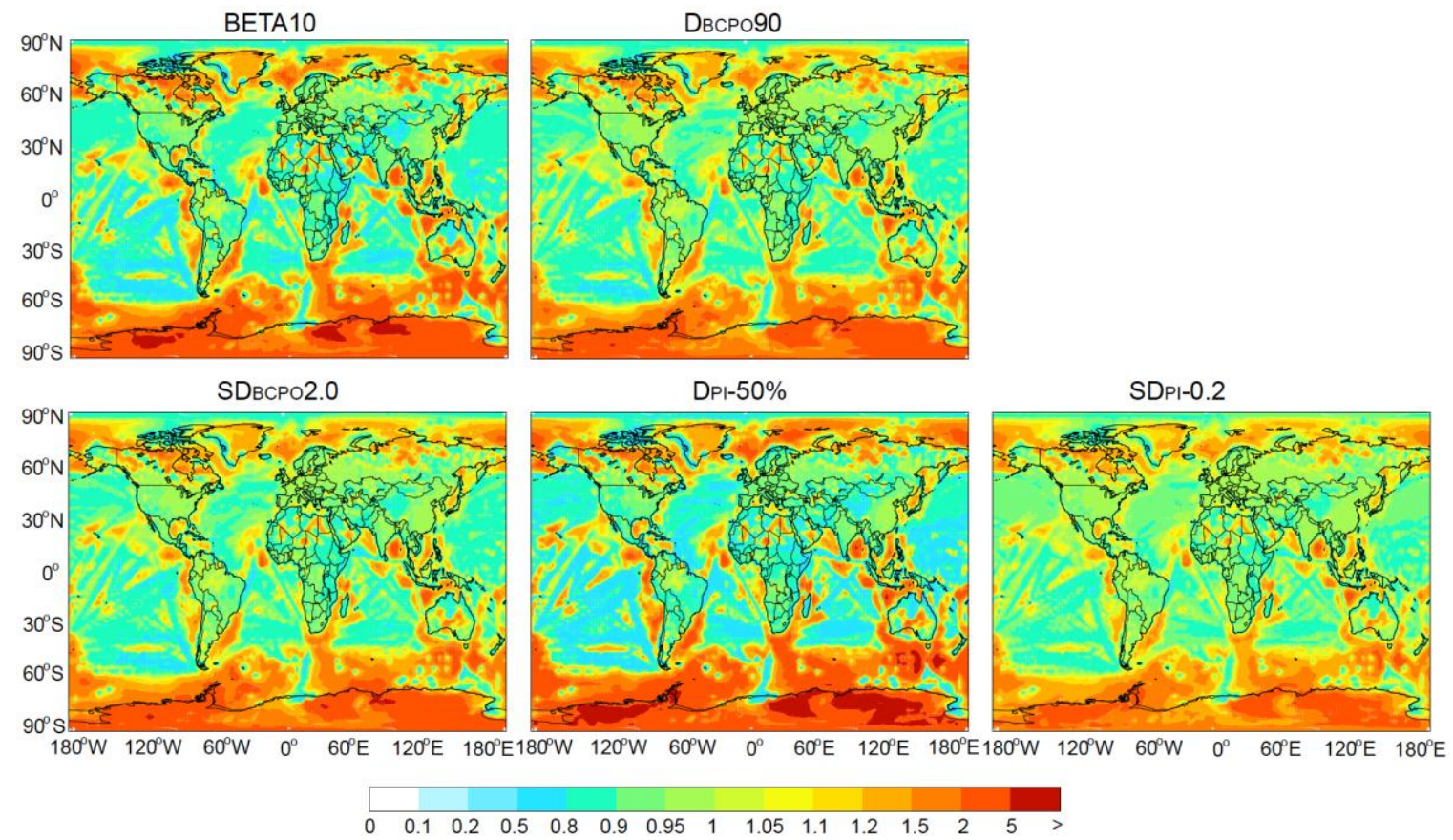

Figure S13. GEOS-Chem simulated ratios of annual mean $\mathrm{BC}$ aging rates from five microphysics-based sensitivity simulations (Table 1) to that from the standard microphysics-based simulation in the surface layer. See Table 1 and text for details. Model results are for 2009. 


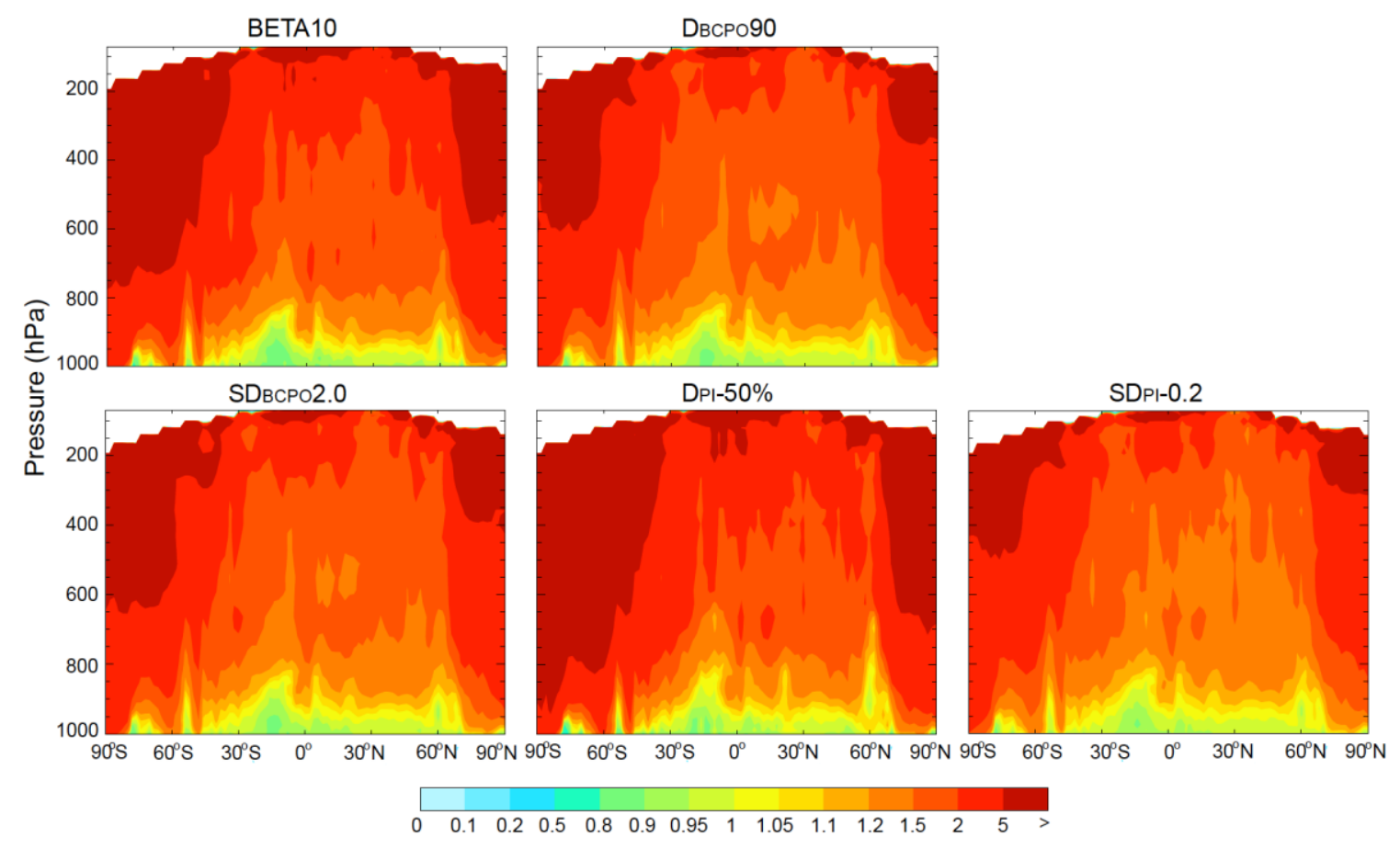

Figure S14. Same as Fig. S13, but for zonal mean ratios. 

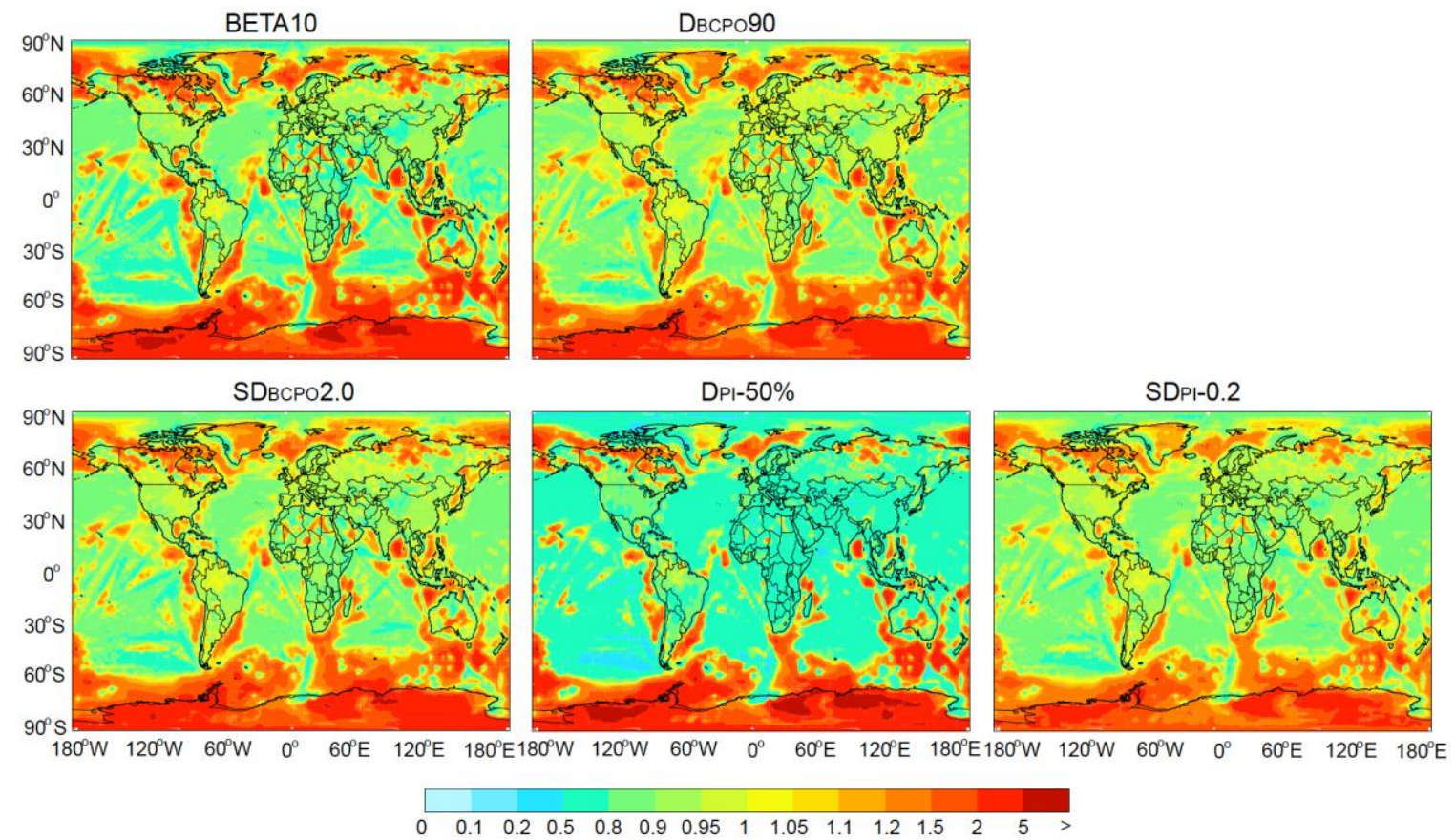

Figure S15. Same as Fig. S13, but for ratios of BC aging rates through condensation. 


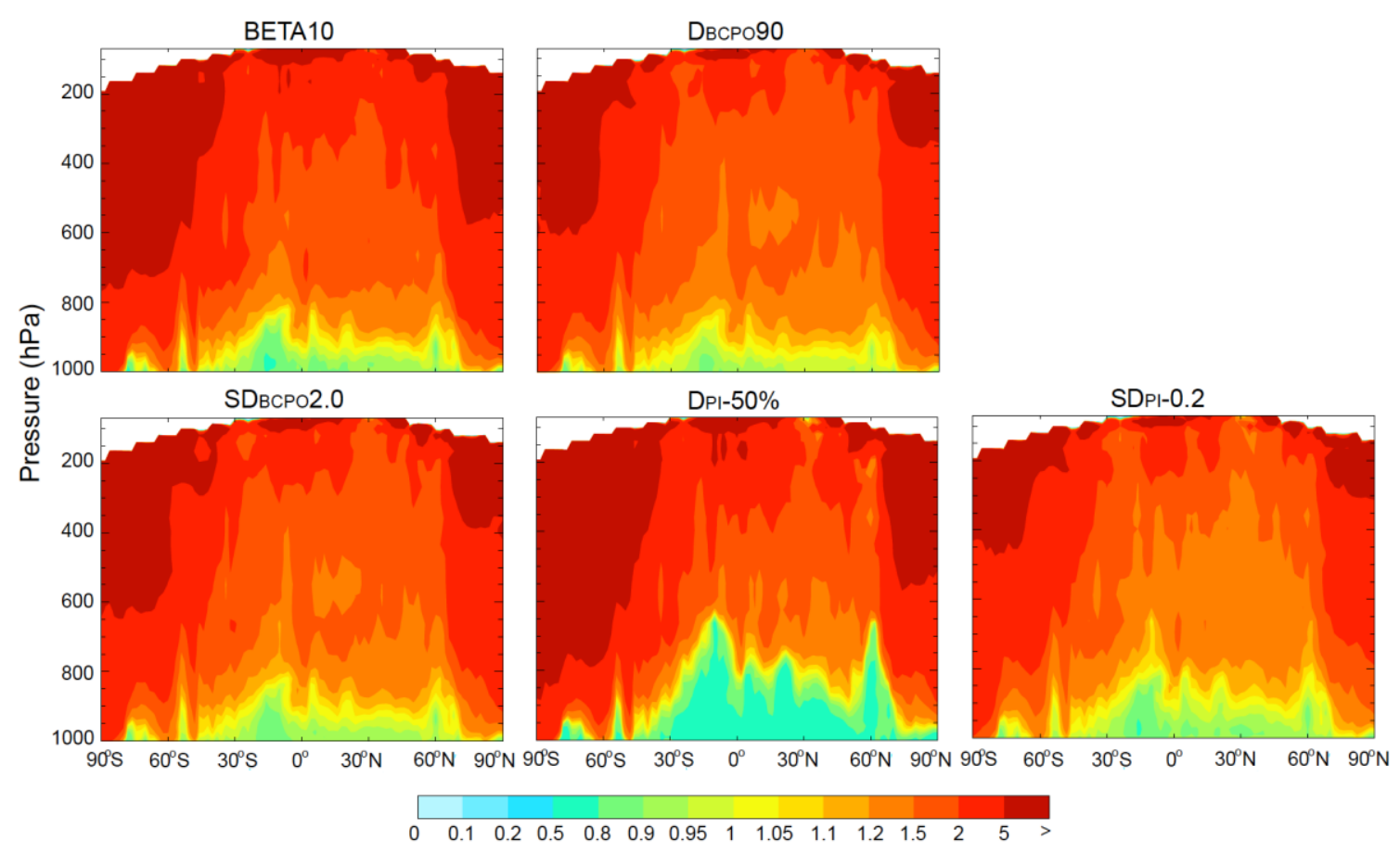

Figure S16. Same as Fig. S15, but for zonal mean ratios. 

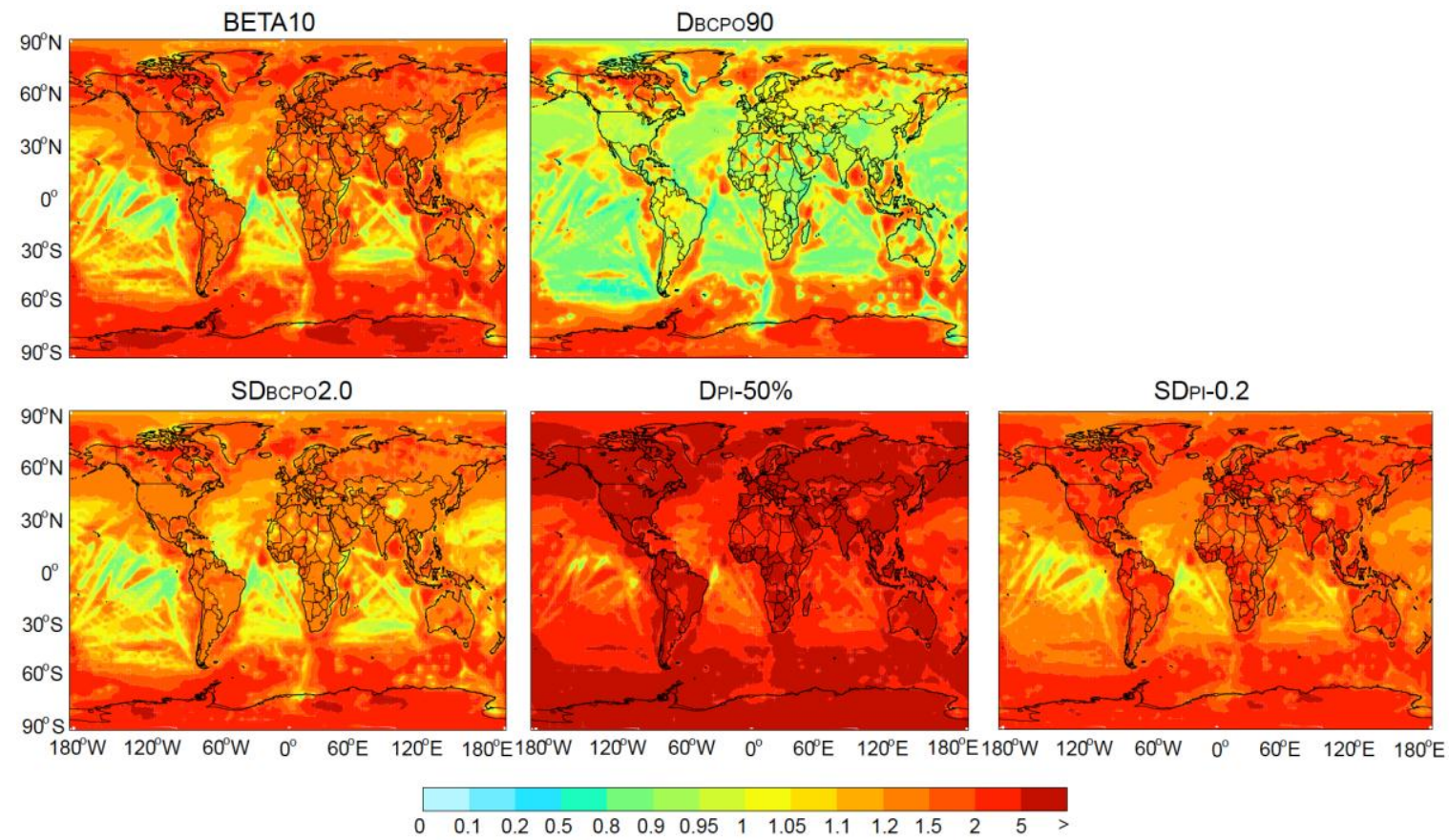

Figure S17. Same as Fig. S13, but for ratios of BC aging rates through coagulation. 


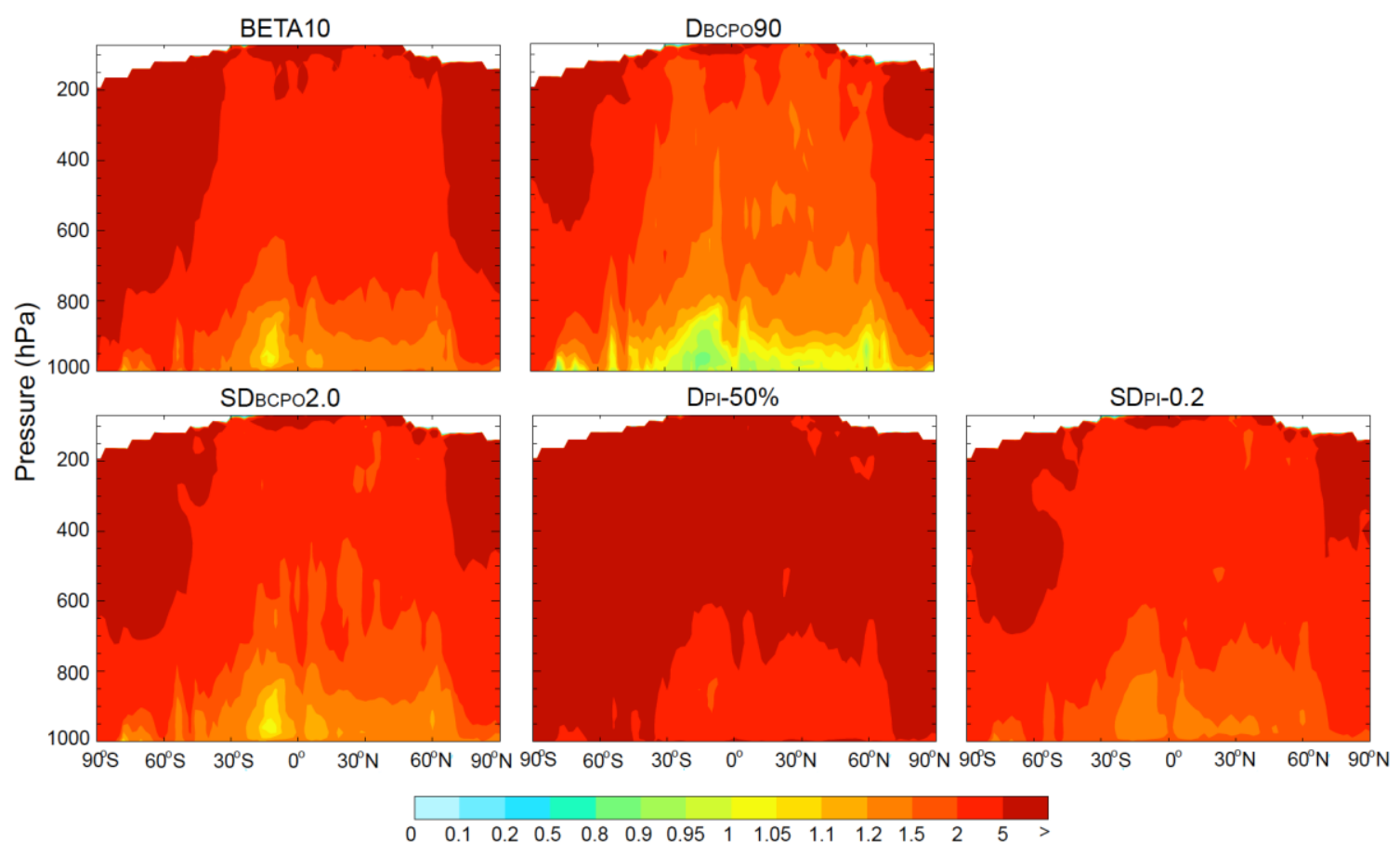

Figure S18. Same as Fig. S17, but for zonal mean ratios. 

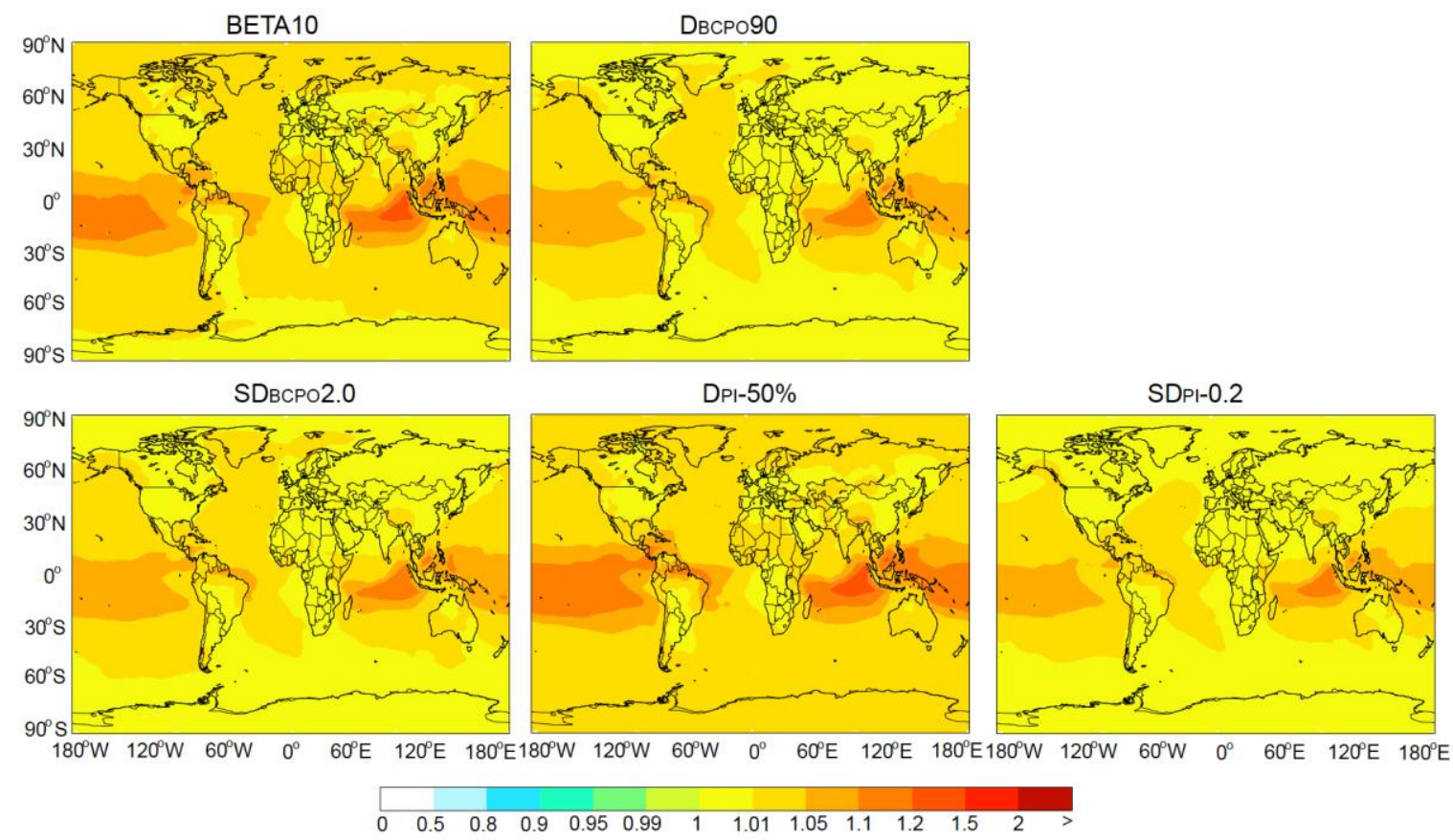

Figure S19. Same as Fig. S13, but for BC column burden. 


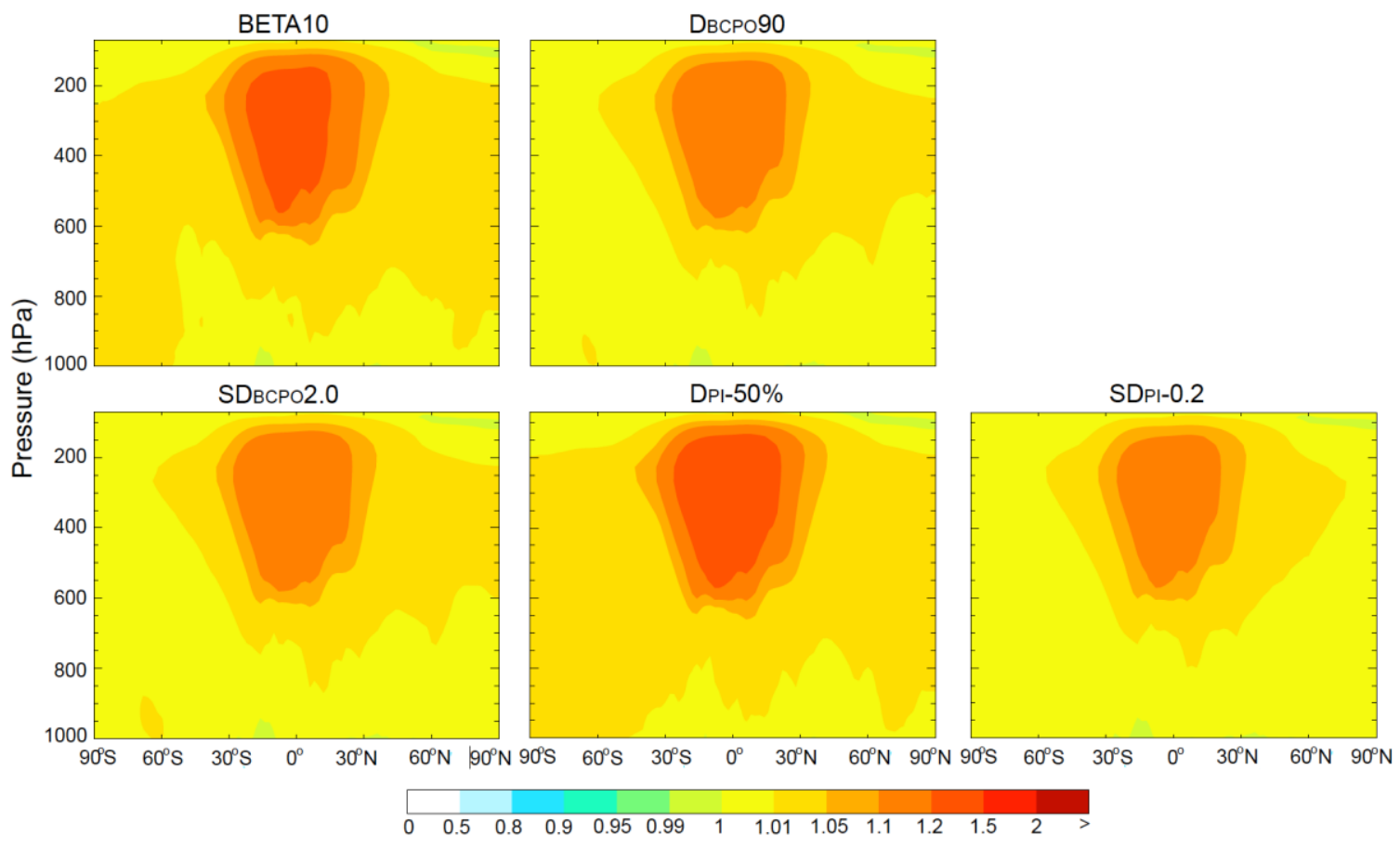

Figure S20. Same as Figure S19, but for zonal mean BC concentrations. 